\title{
Digital Watermarking Algorithm Based on Spread Spectrum and Fourier Transform
}

\author{
Zhanping Huang ${ }^{1}$, a Jinxing $\mathrm{Liu}^{2}, \mathrm{~b}^{*}$, Qing Lv ${ }^{1}, \mathrm{c}$, Hua Zhao ${ }^{1}$ \\ ${ }^{1}$ Hebei Normal University, Shijiazhuang, 050024, China \\ ${ }^{2}$ Hebei University of Science \& Technology, Shijiazhuang, 050080, China \\ ahzp6617287@163.com, b657277728@qq.com, c Ivqing1017@163.com
}

Fund Project: Fund Project of Hebei Department of Education (n2014116), Youth Fund of Hebei Normal University (12010q10), Youth Fund of Hebei Normal University (12015q12), Doctoral Fund of Hebei Normal University (12012b15).

Keywords: Digital watermarking; Fourier transform; Spread spectrum; Sequence pairs; MATLAB simulation

\begin{abstract}
In recent years, computer and the network technology has been developing at breakneck speed, accompanied by a brand new digital era. With the further development of digitalization, an important issue has gradually emerged, that is, the security of digital content. In this context, a new digital technologies-digital watermarking technology is proved. It uses a certain method of embedding digital information hidden in the carrier-specific elements such as images, audio, or video, to determine the authenticity of a digital source, copyright holder information and does not affect the availability of hosted data. There are many kinds of digital watermark embedding and extracting algorithm, in this paper, based on Fourier transform domain Watermarking algorithm. In order to improve the security and robustness of watermarking system, and watermark preprocessing using spread-spectrum technology and use of sequence pairs as PN spread spectrum modulation of the watermark, the spreading sequences generated-code embedded in the DFT transforms the original image, and then through the IDFT transforms images can be watermarked. Watermark is also used in the extraction of sequence pairs on spread spectrum watermark embedding and restores the original image information. Finally was achieved by MATLAB simulation based on DFT-transform domain Watermarking algorithm of sequence spread spectrum and from the experimental results: with the increase of embedding strength, watermarked image distortions become increasingly evident, but to extract the watermark effect is getting better and better. Spread spectrum watermarking technology and engineering simulation for this paper have some reference values.
\end{abstract}

\section{Introduction}

So far, researches of the watermarking technology have achieved great progress both in space domain and frequency domain, and the progressing water technology is gradually meeting people's requirement for information security and copyright protection, etc. However, it is undeniable that with all these achievements, many challenging difficult problems in researches still remain to be solved and the watermarking technology is still immature but remains to be improved. For example, 
Watermark embedding and detection, watermarking capacity of mathematical models, estimation methods, watermarks and reliable way to recover, new applications of digital watermark and so on.

Study on digital watermark technology in the future will focus on new ways to further enhance the security, robustness and resistance to attack, get rid of limitations, and more research into areas such as cryp to graphy, multimedia and signal processing, pattern recognition, a good combination can get better the deeper development of watermarking technology.

In spread-spectrum communication system, the spread-spectrum sequences and its transmitter receiver solution sequences and do not have to be the same sequence, as long as these 2 sequences (called sequence pair) meet certain conditions to meet demands for optimum signal, which is sequence spread spectrum systems with other systems difference. Which leads sequence occasionally of concept: set $\mathrm{x}$ and $\mathrm{y}$ is two a length are for $\mathrm{n}$ of sequence, which: $\mathrm{X}=(\mathrm{x} 0, \mathrm{x} 1, \ldots, \mathrm{xN}-1)$, and $\mathrm{Y}=$ $(\mathrm{y} 0, \mathrm{y} 1, \ldots, \mathrm{yN}-1)$, is said $\mathrm{x}$ and $\mathrm{y}$ composition a $\mathrm{n}$ long of sequence occasionally, remember made $(\mathrm{X}, \mathrm{Y})$; if sequence $\mathrm{x}$, and $\mathrm{y}$ elements take value for plus or minus 1 , is said sequence occasionally $(\mathrm{X}, \mathrm{Y})$ for binary sequence pairs.

It can be known from literature [6,7] that sequence pair, Gold code and $\mathrm{m}$ series as a spread-spectrum sequence, compared to the other two, bit error rate performance of the former is a bit poor, but it can exist in any length, this cover can exist such as Gold series, m series, some fixed-length limitation. And found in the experiment if we can choose the right sequence, when the system has a large sinusoidal interference, its performance may even be better than the Gold series and $\mathrm{m}$ series. Based on the above analysis, we use sequence pairs to spread spectrum Watermarking information processing.

\section{Principles of Watermarking Embedding Algorithm and Realization of MATLAB}

Diagram of Embedding Algorithm's Principles. Diagram of embedding algorithm's principles is as shown in Fig. 1

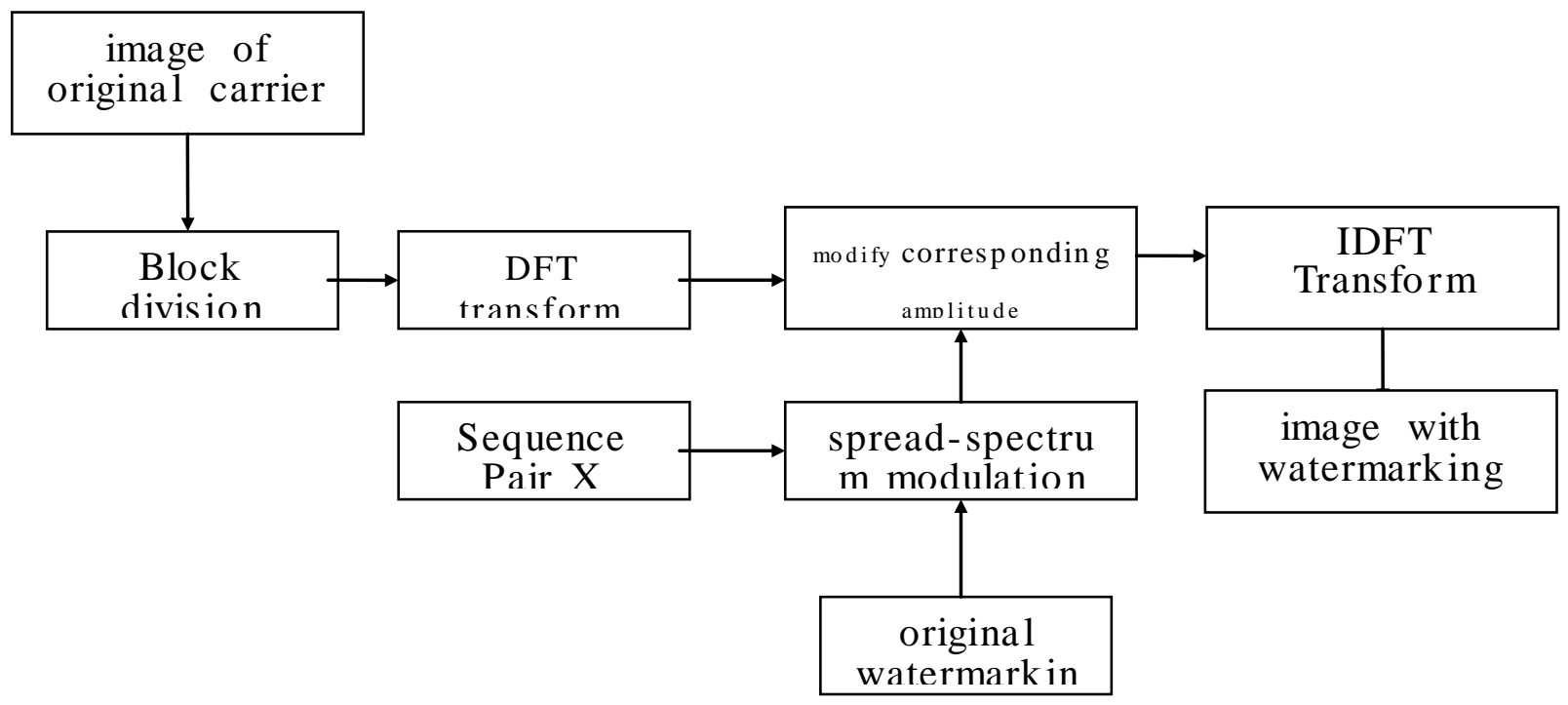

Fig. 1 Diagram of Watermarking Printing's Principles 
First, divide blocks of the original image into $8 * 8$ blocks of data, and then carry out two-dimensional discrete Fourier transform to each data block, followed by pretreatment of the original watermarking information (that is, use sequence pairs for spread spectrum). After all the above works have been completed, the spreading sequences generated code with block segmentation and DFT transformed amplitude spectrum overlay image, this is equivalent to the watermark, after expanding successfully loaded in the original image. Embedding success after the inverse discrete Fourier transform of the image contains a watermark image can be obtained.

Process of Realizing MATLAB for Embedding Algorithm. Step One: reasonably set relevant parameters of embedding, such as strength coefficients and sizes of blocks.

Step Two: use the imread function in the function date to read the original carrier image, and the general format of this function is $A=$ imread (' $D$ :/digital watermarking/yuanshizaitit.bmp') . In addition, use imshow function to show this image and then use imresize function to turn the size of original image into $512 * 512$ pixel.

Step Three: also use imshow function to read the original watermarking image, and use imresize function to turn it into a $64 * 65$ pixel image and show it.

Step Four: Generate the sequence pair X, and then repeat the original watermarking information for 61 times according to its position, that is, length of the sequence pair used in this paper; and then, carry out exclusive spread spectrum towards the sequence $X$ in this sequence pair to generate new spread spectrum sequence code.

Step Five: Divide the original carrier image into $8 * 8$ data block, and then make DFT transform towards each data block, that is, use the $\mathrm{fft} 2$ function to transform the carrier information to the spectrum, and then, add spectrum watermarking information correspondingly on the magnitude spectra.

Step Six: use ifft 2 function to make the Fourier inverse transform towards toe carrier image embedded with watermarking formation to get carrier image with watermarking information and show it.(for source code of embedding algorithm, refer to the attachment)

\section{Principle of Watermarking Extraction Algorithm and Realization of MATLAB}

Diagram of Extraction Algorithm's Principles. Diagram of extraction algorithm's principle is as shown in Fig. 2

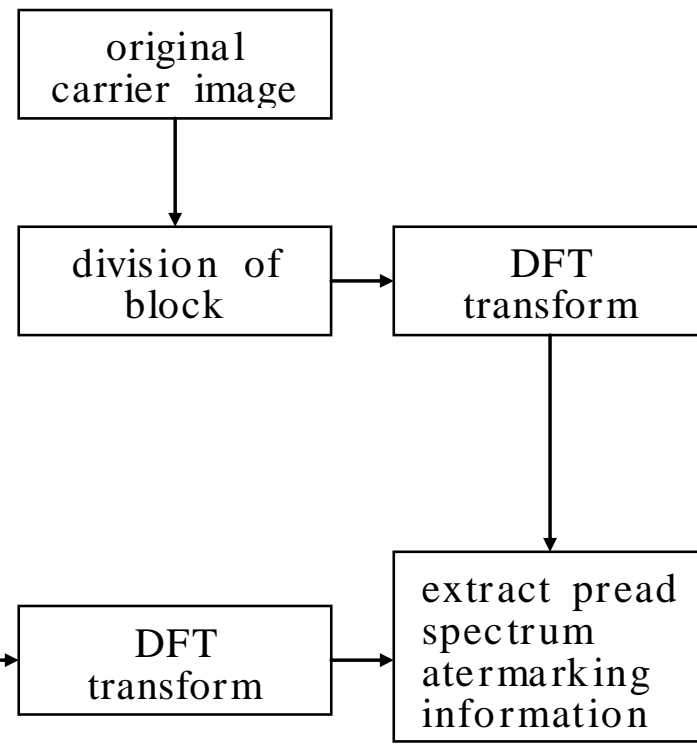

Fig. 2 Diagram of Watermarking Extraction

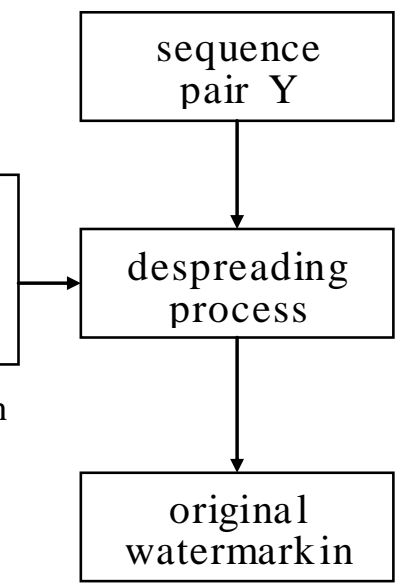


This algorithm uses the embedding algorithm for similar steps in the previous article, it is already contains a watermark of vector images into different blocks, then for each sub-block DFT, DFT transform and thereafter after block with the same amplitude comparison of the original image, thus effectively spread spectrum Watermarking information. Completed the steps above and then use the sequence pair Y on spread-spectrum despreading information extracted so that you get the original image information.

MATLAB Realization Process of Extracting Algorithm. Step One: Read carrier image and original carrier image with watermarking information and show them respectively with imshow function.

Step Two: Divide blocks of carrier image and original image with watermarking information and make corresponding transform with $\mathrm{fft} 2$ function.

Step Three: compare the information amplitudes of two images obtained in step three so as to extract information of spread spectrum watermarking, and the specific extraction rules are: of amplitude of the previous one is larger than the later, it indicates that code value in the spread spectrum sequence used before is 1 , otherwise the code value is 0 .

Step Four: Despreading, that is, in a dual symbol sequence length and sequence spread spectrum watermark information bit by bit multiplying even $\mathrm{Y}$, and then sequence despread symbol period is evaluated within Canada, and with the decision threshold comparison, since the sequence pairs have negative correlation opposite, so if the comparison result is greater than 0 the answer is zero, if the comparison result is less than 0 sentenced to 1 .

Step Five: show watermarking image that has been extracted with imshow function.

(for extraction of algorithm's source code, refer to the attachment)

\section{Simulation Results and Analysis}

Results of the simulation program are shown in Fig. 4, Fig. 5. Fig. 6, Fig. 7, Fig. 8 and Fig. 9.

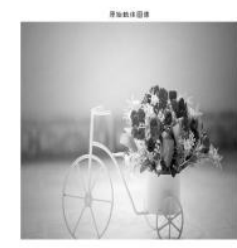

Fig. 4 Original Carrier Image

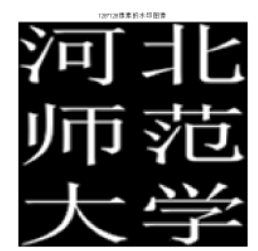

Fig. 5

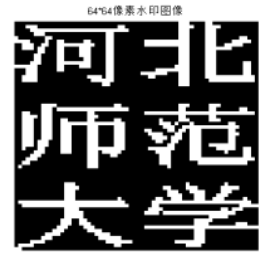

Fig. $6 \quad 64 * 64$ Watermarking Image

序列偶扩频后得到的水印信息

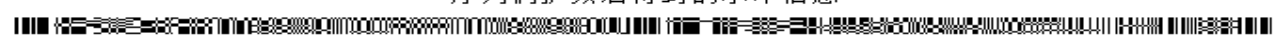

Fig. 7 Watermarking Information after Spreading Sequence Pairs

Change the size of embedding strength $\mathrm{k}$, and make it as $0.015,0.2,0.025$ and 0.03 respectively. Operate the program and calculate the value of $P S N R$ and $N C$. Results are as follows: 


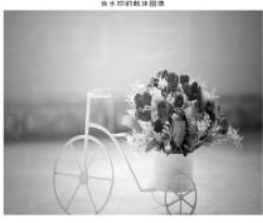

Fig. 8 Carrier Image with Watermarking

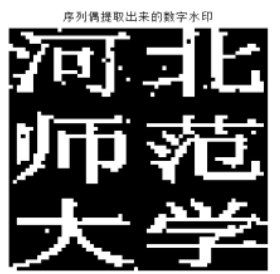

When the embedding strength $\mathrm{k}=0.015 \quad \mathrm{PSNR}=93.522 \quad \mathrm{NC}=0.98013$, images that are produced are as shown in Fig. 10 and Fig. 11.

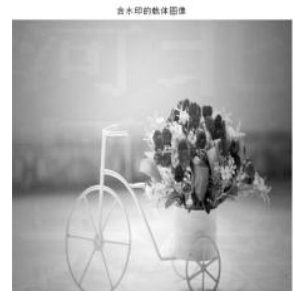

Fig. 10 Carrier Image with Watermarking

When the embedding strength $\mathrm{k}=0.02$ produced are as shown in Fig. 12 and Fig. 13.

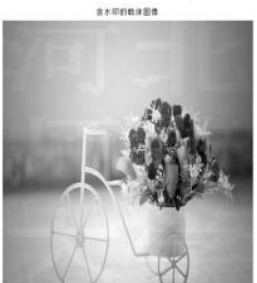

Fig. 12 Carrier Image with Watermarking

When the embedding strength $\mathrm{k}=0.025$ PSNR=83.119 produced are as shown in Fig. 14 and Fig. 15.

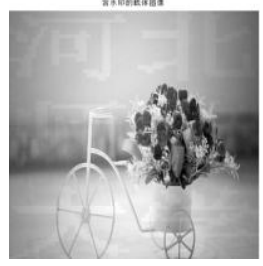

Fig. 14 Carrier Image with Watermarking Fig. 16 Carrier Image with Watermarking Image

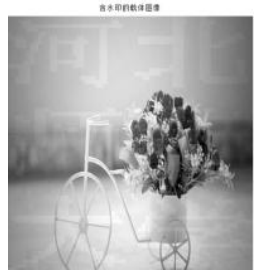

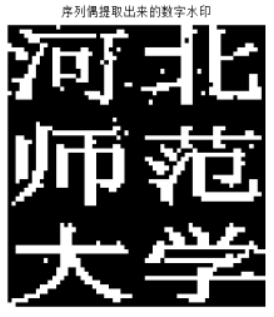

Fig. 11 Extracted Watermarking Image $\mathrm{PSNR}=87.671 \mathrm{NC}=0.98279$, images that are

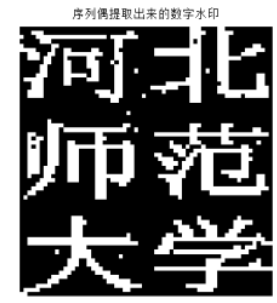

Fig. 13 Extracted Watermarking Image $\mathrm{NC}=0.98281$, images that are Fig. 15 Extra Fig. 17 Extracted Watermarking
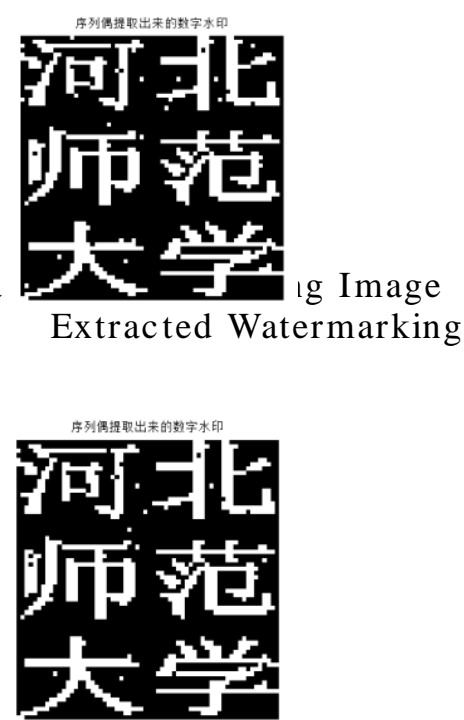

When the embedding strength $\mathrm{k}=0.03 \mathrm{PSNR}=79.385 \mathrm{NC}=0.98409$, images that are produced are as shown in Fig. 16 and Fig. 17. 


\section{Conclusions}

This paper mainly researches digital watermarking algorithm based non spread spectrum and Fourier transform, introduces relevant theoretical knowledge and design new watermarking algorithm as well as adds new design thoughts compared with the existing watermarking algorithm. For example, use sequence pairs as the spread spectrum sequence and combines it with DFT algorithm. Although DFT method's resistance to compression is relatively weak, as we have divided signals into corresponding values and amplitude information, it has more ample detailed information. Currently, there are few water algorithms that have been realized by DFT, so we hope this paper would make some contributions to future researches in this regard. 